\title{
On the upper and lower chromatic numbers of $\operatorname{BSQSs}(16)$ *
}

\author{
Giovanni Lo Faro \\ Department of Mathematics, University of Messina \\ Salita Sperone, 31 - 98166 Sant'Agata - Messina, Italy. \\ E-mail: lofaro@www.unime.it \\ Lorenzo Milazzo \\ Department of Mathematics, University of Catania \\ Viale A. Doria, 695125 - Catania, Italy. \\ E-mail: milazzo@dipmat.unict.it \\ Antoinette Tripodi \\ Department of Mathematics, University of Messina \\ Salita Sperone, 31 - 98166 Sant'Agata - Messina, Italy. \\ E-mail: tripodi@dipmat.unime.it
}

Submitted: September 6, 1999; Accepted: October 20, 2000

\begin{abstract}
A mixed hypergraph is characterized by the fact that it possesses $\mathcal{C}$-edges as well as $\mathcal{D}$-edges. In a colouring of a mixed hypergraph, every $\mathcal{C}$-edge has at least two vertices of the same colour and every $\mathcal{D}$-edge has at least two vertices coloured differently. The upper and lower chromatic numbers $\bar{\chi}, \chi$ are the maximum and minimum numbers of colours for which there exists a colouring using all the colours. The concepts of mixed hypergraph, upper and lower chromatic numbers are applied to SQSs. In fact a BSQS is an SQS where all the blocks are at the same time $\mathcal{C}$-edges and $\mathcal{D}$-edges. In this paper we prove that any $B S Q S(16)$ is colourable with the upper chromatic number $\bar{\chi}=3$ and we give new information about the chromatic spectrum of BSQSs(16).
\end{abstract}

*Supported by cofin. MURST "Strutture geometriche, combinatorie e loro applicazioni" and by C.N.R. (G.N.A.S.A.G.A.). 


\section{Introduction}

A mixed hypergraph $[11,12]$ is a triple $\mathcal{H}=(X, \mathcal{C}, \mathcal{D})$, where $X$ is a finite set of vertices, while $\mathcal{C}$ and $\mathcal{D}$ are two families of subsets of $X$. The elements of $\mathcal{C}$ and $\mathcal{D}$ are called $\mathcal{C}$-edges and $\mathcal{D}$-edges respectively. If $\mathcal{C}=\emptyset$ then $\mathcal{H}$ is called a $\mathcal{D}$-hypergraph, while if $\mathcal{D}=\emptyset$ then $\mathcal{H}$ is called a $\mathcal{C}$-hypergraph.

A strict $k$-colouring of $\mathcal{H}$ is a vertex colouring where any $\mathcal{C}$-edge has at least two vertices of the same colour and any $\mathcal{D}$-edge has at least two vertices coloured differently, and exactly $k$ colours are used in it. If it is not necessary to know the number of used colours then a strict k-colouring will be called strict colouring.

The minimum (maximum) $k$ for which there exists a strict k-colouring is called the lower (upper) chromatic number of $\mathcal{H}$ and is denoted by $\chi(\bar{\chi})$. If there exists no strict colouring of $\mathcal{H}$, then $\mathcal{H}$ is said to be uncolourable.

Two strict colourings of $\mathcal{H}$ are different if there exist two vertices such that in one colouring they have different colours and with the same colour in the other one. Let us denote $r_{k}$ as the number of different strict colourings of $\mathcal{H}$ using $k$ colours; we will call the vector $R(\mathcal{H})=\left(0,0, \ldots, r_{\chi}, \ldots, r_{\bar{\chi}}, \ldots, 0,0\right)$ the chromatic spectrum of $\mathcal{H}[12,6]$.

The set $L \subseteq X$ is called a $\mathcal{C}$-stable set $(\mathcal{D}$-stable set) if it does not contain any $\mathcal{C}$-edges ( $\mathcal{D}$-edges). If $L$ does not contain $\mathcal{C}$-edges and $\mathcal{D}$-edges at the same time then it is called a bi-stable set.

A $\mathcal{D}$-hypergraph is a classical hypergraph [1] and its lower chromatic number is the chromatic number introduced by Erdős and Hajnal in 1966 [3].

In this paper the concepts of strict colouring and upper and lower chromatic numbers are applied to a particular t-design called a Steiner quadruple system.

Briefly, by a $t$-design $S_{\lambda}(t, k, v)$ we mean a pair $(X, B)$ where $X$ is a $v$-set of vertices and $B$ is a collection of k-element subsets of $X$, usually called blocks such that every $t$-element subset of $X$ occurs in exactly $\lambda$ blocks of $B$. When $\lambda=1$ the $t$-design is referred to as a Steiner system $S(t, k, v)$.

A Steiner Quadruple System (briefly SQS) is an $S(3,4, v)$. In 1960 Hanani [5] proved that an $S Q S(v)$ exists if and only if $v \equiv 2$ or $4 \quad(\bmod 6)$.

A $B S Q S$ is a Steiner Quadruple System where all the blocks are at the same time $\mathcal{C}$-edges and $\mathcal{D}$-edges [8].

In $[8,9,10]$ the necessary conditions for the existence of strict colourings for $B S Q S$ s were determined and the exact value of the upper chromatic number for $B S Q S(8), B S Q S(10)$ was found in [9].

In [9] it was proved that for $B S Q S s(16)$ obtained by doubling construction $\bar{\chi}=3$ or they are uncolourable.

The aim of this paper is to prove that any $B S Q S(16)$ is colourable with $\bar{\chi}=3$ and we will show that only two kinds of chromatic spectrum can exist for a BSTS(16). 
In the next section we will use the following theorem, proved in [4] for more general Steiner systems than SQSs.

Theorem 1 In an $S Q S(v)(X, B)$, if $H \subseteq X,|H|=h, T_{H}$ is the set of blocks included in $H$, and $T_{X-H}$ is the set of blocks included in $X-H$, then

1. $f(v, h)=\left|T_{H}\right|-\left|T_{X-H}\right|, f(v, h)=b_{0}-\left(\begin{array}{c}v-h \\ 1\end{array}\right)=b_{1}+\left(\begin{array}{c}v-h \\ 2\end{array}\right) b_{2}-\left(\begin{array}{c}v-h \\ 3\end{array}\right)$, where $b_{i}=\left(\begin{array}{c}v-i \\ 3-i\end{array}\right) /\left(\begin{array}{c}4-i \\ 3-i\end{array}\right)$ for $0 \leq i \leq 2$;

2. If $d_{H}(x)=\mid\{b \in B: x \in b$ and $b \subseteq H\} \mid$ and $\delta_{H}(x)=\mid\{b \in B: x \in b-\{x\}$ and $b \subseteq X-H\} \mid$, then

$$
d_{H}(x)+\delta_{H}(x)=f(v, h)-f(v, h-1) .
$$

\section{Upper chromatic number of BSQS(16)}

Let us assume that a BSQS is colourable with the strict colouring $\mathcal{P}$ which uses $h$ colours, the colouring class $X_{i}$ is the vertex set coloured with the colour $(i)$ and $\left|X_{i}\right|=n_{i}$. It is clear that $\mathcal{P}$ partitions the vertex set into a family of bi-stable sets. Let us consider the class of strict k-colourings which partition the vertex set into $k$ colouring classes with cardinalities $n_{1}, n_{2}, \cdots, n_{k}$. We will denote this class with the $h$-tuple $\left(n_{1}, n_{2}, \cdots, n_{h}\right)$ where $n_{i} \leq n_{i+1}$ for $1 \leq i \leq h$. $\mathcal{P}$ colours the blocks of BSQS in three possible ways:

i) Three vertices are coloured with one colour and the other vertex is coloured with another one;

ii) Two vertices are coloured with one colour and the other two vertices are coloured with another one;

iii) Two vertices are coloured with one colour and the other two vertices are coloured with two different colours different from the first.

If $I \subseteq\{1,2, \cdots, h\}$, with $|I| \geq 2$, then let us define the set of vertices $\mathcal{S}_{I} \subseteq X$ as the union of $|I|$ colouring classes coloured with the colours (i) inside $I$. The number of triples of vertices coloured with different colours of $\mathcal{P}$ in $\mathcal{S}_{I}$, if $\left|\mathcal{S}_{I}\right|=s_{I}$, is:

$$
c_{I}=\left(\begin{array}{c}
s_{I} \\
3
\end{array}\right)-\left[\sum_{j \in I}\left(\begin{array}{c}
n_{j} \\
3
\end{array}\right)+\sum_{j \in I}\left(\begin{array}{c}
n_{j} \\
2
\end{array}\right)\left(s_{I}-n_{j}\right)\right]
$$


by the definitions of strict colouring and SQS we can give the following proposition.

Proposition 1 If $\mathcal{P}$ is a strict colouring for a BSQS which uses $h$ colours, then 1) $\sum_{i=1}^{h}\left(\begin{array}{c}n_{i} \\ 3\end{array}\right)$ blocks are coloured as in i);

2) $\left(c_{I^{\prime}} / 2\right)$ blocks are coloured as in iii), where $I^{\prime}=\{1,2, \cdots, h\}$;

3) $|B|-\frac{c_{I^{\prime}}}{2}-\sum_{i=1}^{h}\left(\begin{array}{c}n_{i} \\ 3\end{array}\right)$ blocks are coloured as in $\left.i i\right)$;

4) all the $c_{I}$ have to be even.

The following theorem proves that any BSQS(16) is uncolourable with four colours.

Theorem 2 If a BSQS(16) is colourable then $\bar{\chi} \leq 3$.

\section{Proof.}

In [9] it was proved that $\bar{\chi} \leq 4$ and if a BSQS(16) is colourable with four colours then it is necessary to use one of the following strict colourings: $p=(2,4,5,5)$ or $k=(2,3,5,6)$. We will prove that the colourings $p$ and $k$ are not strict colourings and $\bar{\chi}=3$. Let $X=\{1,2, \ldots, 16\}$ be the set of vertices of $B S Q S(16)$.

If $p=(2,4,5,5)$ is a strict colouring, by theorem 1 we have $\left|T_{X_{1} \cup X_{2} \cup X_{3}}\right|=25$. However, the number of 3-chromatic blocks coloured with the colours (1), (2) and (3) is 20 and the number of bi-chromatic blocks coloured with the colours (2) and (3) is at least 7 and so $\left|T_{X_{1} \cup X_{2} \cup X_{3}}\right| \geq 27$ and this is absurd.

Let us consider a strict colouring $k=(2,3,5,6)$.

We have $\left|T_{X_{1} \cup X_{2} \cup X_{3}}\right|=15$, so all the blocks of $T_{X_{1} \cup X_{2} \cup X_{3}}$ are 3-chromatic. It is important to note that a monochromatic pair of vertices coloured with the colour (1), (2) and (3) is present at least once in the blocks of $T_{X_{1} \cup X_{2} \cup X_{3}}$, and precisely 13 of the 14 above-mentioned monochromatic pairs are present exactly once while the remaining pair is present twice.

Let us define the colouring classes in this way: $X_{1}=\{1,2\}, X_{2}=\{3,4,5\}, X_{3}=$ $\{6,7,8,9,10\}$ and $X_{4}=\{11,12,13,14,15,16\}$. All the blocks of $T_{X_{1} \cup X_{2} \cup X_{3}}$ contain the $\{1\}$ vertex or the $\{2\}$ vertex; more precisely: seven blocks contain $\{1\}$ and not $\{2\}$; seven blocks contain $\{2\}$ and not $\{1\}$ and exactly one block contains the pair $\{1,2\}$. We may assume that $\{1,2,3,6\} \in T_{X_{1} \cup X_{2} \cup X_{3}}$ and that the pair $\{3,4\}$ appears in a block containing $\{1\}$, so without loss of generality we can share the blocks of $T_{X_{1} \cup X_{2} \cup X_{3}}$ in the following way: 


$\begin{array}{ll}\{1,2,3,6\} & \{2,3,8,9\} \\ \{1,3,4,7\} & \{2,3,7,10\} \\ \{1,3,5,8\} & \{2,4,5, \cdot\} \\ \{1,3,9,10\} & \{2,4, \cdot \cdot \cdot\} \\ \{1,4,6,9\} & \{2,4, \cdot \cdot \cdot\} \\ \{1,4,8,10\} & \{2,5, \cdot \cdot \cdot\} \\ \{1,5,7,9\} & \{2,5, \cdot \cdot \cdot\} \\ \{1,5,6,10\} & \end{array}$

Table 1

We cannot add the pairs $\{6,7\},\{6,8\}$ and $\{7,8\}$ inside the incomplete blocks of Table 1 without violating the definition of SQS.

The next theorem will prove that all BSQSs(16) are colourable with strict colourings which use three colours. This theorem is important because it shows that uncolourable $\operatorname{BSQSs}(16)$ do not exist.

Theorem 3 For any $B S Q S(16), \bar{\chi}=3$.

\section{Proof.}

Let $(S, \mathcal{B})$ be a $B S Q S(16)$. It is not difficult to see that we can find a bi-stable set $N_{1}$ with $\left|N_{1}\right|=6$. Let us assume $N_{1}=\{1,2,3,4,5,6\}$. If $H=\{7,8,9, \ldots, 16\}$ then $\left|T_{H}\right|-\left|T_{N_{1}}\right|=15$ and so $\left|T_{H}\right|=15$.

Let $h_{1} \in H$ be such that $d_{H}\left(h_{1}\right)=\max \left\{d_{H}(h): h \in H\right\}$ and let us assume $h_{1}=7$. By 2 . of theorem 1 we have $d_{H}(7)=6+x$, with $0 \leq x \leq 2$. If $H^{\prime}=H-\{7\}$ then $\left|T_{H^{\prime}}\right|=9-x$. Let $h_{2} \in H^{\prime}$ such that $d_{H^{\prime}}\left(h_{2}\right)=\max \left\{d_{H^{\prime}}(h): h \in H^{\prime}\right\}$ and suppose $h_{2}=8$. We have $d_{H^{\prime}}(8) \geq \frac{4(9-x)}{9}=4-\frac{4}{9} x$. Let $d_{H^{\prime}}(8)=4+y$. The pair $\{7,8\}$ is obviously contained in at least one block of $T_{H}$ and so $0 \leq y \leq 3$. Let us denote with $\mathcal{B}^{\star}$ the set of blocks $b$ of $T_{H}$ such that $b \cap\{7,8\}=\emptyset$, obviously $\left|\mathcal{B}^{\star}\right|=5-(x+y)$. Let us consider the following cases.

Case 1: $x+y=5$. The sets of vertices $N_{1},\{7,8\},\{9,10, \ldots, 16\}$ give a strict colouring for $B S Q S(16)$.

Case 2: $x+y=4$. If $b=\{9,10,11,12\}$ is the only block of $\mathcal{B}^{\star}$, then $N_{1},\{7,8,9\}$, $\{10,11, \ldots, 16\}$ give a strict colouring for $B S Q S(16)$.

Case 3: $x+y=3$. Let $b_{1}, b_{2}$ be the blocks of $\mathcal{B}^{\star}$. If $b_{1} \cap b_{2}=\emptyset$, then we can assume that $b_{1}=\{9,10,11,12\}$ and $b_{2}=\{13,14,15,16\}$. Let $\{7,8,9, k\} \in \mathcal{B}$, then it is possible 
to find $t \in b_{2}, t \neq k$, such that $N_{1},\{7,8,9, t\},\{10,11, \ldots, 16\}-\{t\}$ give a strict colouring for $B S Q S(16)$.

Case 4: $x+y=2$. Let $\mathcal{B}^{\star}=\left\{b_{1}, b_{2}, b_{3}\right\}$. We can assume that there exists an element, let us say 9 , such that $9 \in b_{1} \cap b_{2}$. If $9 \in b_{3}$ then $N_{1},\{7,8,9\}$ and $\{10,11, \ldots, 16\}$ give a strict colouring for $B S Q S(16)$; otherwise, let $b_{3}=\{10,11,12,13\}$ and $\{7,8,9, k\} \in \mathcal{B}$, then it is possible to find $t \in b_{3}, t \neq k$, such that $N_{1},\{7,8,9, t\},\{10,11, \ldots, 16\}-\{t\}$ give a strict colouring for $B S Q S(16)$.

Case 5: $x+y=1$. Let $\mathcal{B}^{\star}=\left\{b_{1}, b_{2}, b_{3}, b_{4}\right\}$. If $b_{1} \cap b_{2} \cap b_{3} \cap b_{4} \neq \emptyset$ (let us assume $\left.9 \in b_{1} \cap b_{2} \cap b_{3} \cap b_{4}\right)$ then $N_{1},\{7,8,9\},\{10,11, \ldots, 16\}$ give a strict colouring for $B S Q S(16)$. If $b_{1} \cap b_{2} \cap b_{3} \cap b_{4}=\emptyset$, then we have two possibilities.

i) There are three blocks of $\mathcal{B}^{\star}$, let us say $b_{1}, b_{2}, b_{3}$, such that $b_{1} \cap b_{2} \cap b_{3} \neq \emptyset$. We can assume that $9 \in b_{1} \cap b_{2} \cap b_{3} b_{4}=\{10,11,12,13\}$. If $\{7,8,9, k\} \in \mathcal{B}$, then it is possible to find $t \in b_{4}, t \neq k$, such that $N_{1},\{7,8,9, t\},\{10,11, \ldots, 16\}-\{t\}$ give a strict colouring for $B S Q S(16)$.

ii) For every $z \in\{9,10, \ldots, 16\}$ there are exactly two blocks of $\mathcal{B}^{\star}$ containing $z$. Then we can assume that

$$
\begin{gathered}
b_{1}=\{9, \cdot, \cdot, \cdot\}, \quad b_{2}=\{9, \cdot, \cdot, \cdot\} \\
b_{3}=\{10, \cdot, \cdot, \cdot\}, \quad b_{4}=\{10, \cdot, \cdot, \cdot\}
\end{gathered}
$$

If $\{7,8,9,10\} \notin \mathcal{B}$, then $N_{1},\{7,8,9,10\},\{11,12, \ldots, 16\}$ give a strict colouring for $B S Q S(16)$. Let $\{7,8,9,10\} \in \mathcal{B}$, we can assume, without loss of generality, that

$$
\begin{aligned}
& b_{3}=\{10,11,12,13\} \\
& b_{4}=\{10,14,15,16\}
\end{aligned}
$$

and so,

$$
\begin{aligned}
& b_{1}=\{9,11,12,14\} \\
& b_{2}=\{9,13,15,16\} .
\end{aligned}
$$

If $\{7,8,11,15\} \notin \mathcal{B}$, then $N_{1},\{7,8,11,15\},\{9,10,12,13,14,16\}$ give a strict colouring for $B S Q S(16)$; otherwise $N_{1},\{7,8,11,16\},\{9,10,12,13,14,15\}$ give a strict colouring for $B S Q S(16)$.

Case 6: $x+y=0$. From the hypothesis on $d_{7}$ and $d_{8}$ we have:

1. $d_{z}=6$, for $\forall z \in H$; 
2. any pair of distinct elements of $H$ is contained exactly in two blocks of $T_{H}$.

So $T_{H}$ is one of the three nonisomorphic $S_{2}(2,4,10)$ designs [7].

\begin{tabular}{lll}
\multicolumn{1}{c}{$I$} & \multicolumn{1}{c}{$I I$} & \multicolumn{1}{c}{$I I I$} \\
$\{7,8,9,10\}$ & $\{7,8,9,10\}$ & $\{7,8,9,10\}$ \\
$\{7,8,11,12\}$ & $\{7,8,11,12\}$ & $\{7,8,11,12\}$ \\
$\{7,9,11,13\}$ & $\{7,9,11,13\}$ & $\{7,9,13,14\}$ \\
$\{7,10,14,15\}$ & $\{7,10,14,15\}$ & $\{7,10,15,16\}$ \\
$\{7,12,14,16\}$ & $\{7,12,14,15\}$ & $\{7,11,13,15\}$ \\
$\{7,13,15,16\}$ & $\{7,13,15,16\}$ & $\{7,12,14,16\}$ \\
$\{8,9,14,15\}$ & $\{8,9,14,16\}$ & $\{8,9,15,16\}$ \\
$\{8,10,13,16\}$ & $\{8,10,11,16\}$ & $\{8,10,13,14\}$ \\
$\{8,11,14,16\}$ & $\{8,11,14,15\}$ & $\{8,11,14,16\}$ \\
$\{8,12,13,15\}$ & $\{8,12,13,15\}$ & $\{8,12,13,15\}$ \\
$\{9,10,12,16\}$ & $\{9,10,12,15\}$ & $\{9,10,11,12\}$ \\
$\{9,11,15,16\}$ & $\{9,11,15,16\}$ & $\{9,11,14,15\}$ \\
$\{9,12,13,14\}$ & $\{9,12,13,14\}$ & $\{9,12,13,16\}$ \\
$\{10,11,12,15\}$ & $\{10,11,12,16\}$ & $\{10,11,13,16\}$ \\
$\{10,11,13,14\}$ & $\{10,11,13,14\}$ & $\{10,12,14,15\}$
\end{tabular}

In any case $N_{1},\{7,8,9,11,14\},\{10,12,13,15,16\}$ give a strict colouring for these $B S Q S s(16)$.

In [2] it is shown that it is possible to construct BSQSs(16) which contain blocking sets and BSQSs(16) which do not contain any blocking set. So BSQSs(16) colourable with strict colourings which use two and three colours exist and only "3-strict" colourable $\operatorname{BSQSs}(16)$ also exist.

There are two classes of BSQS(16): the first class contains $\operatorname{BSQSs}(16)$ with $\chi=\bar{\chi}=3$; the second one contains $\operatorname{BSQSs}(16)$ with $\chi=2$ or $\bar{\chi}=3$.

The next theorem completes theorem 3 and gives important information about the chromatic spectrum of BSQSs(16).

Theorem 4 The upper chromatic number for all BSQSs(16) is $\bar{\chi}=3$; the lower chromatic number for a BSQS(16) can be $\chi=3$ or $\chi=2$. 


\section{References}

[1] C. Berge: Hypergraphs: combinatories of finite sets. North Holland, (1989).

[2] J. Doyen, M. Vandersavel: Non isomorphic Steiner quadruple systems. Bullettin de la Société Mathématique de Belgique 13, (1971), 393-410.

[3] P. Erdős, A. Hajnal: On chromatic number of graphs and set-systems. Acta Math. Acad. Sci. Hung. 17, (1966), 61-99.

[4] M. Gionfriddo, G. Lo Faro: 2-colourings in $\mathrm{S}(t, t+1, v)$. Discrete Math. 111, (1993), 263-268.

[5] H. Hanani: On quadruple systems. Canadian Journal Mathematics 12, (1960).

[6] T. Jiang, D. Mubayi, Zs. Tuza, V. Voloshin, D. West: The chromatic spectrum is broken. 6th Twente Workshop on Graphs and Combinatorial Optimization, 2628 May, 1999, H.J. Broersma, U. Faigle and J.L. Hurink (eds.).University of Twente, May, 1999, 231-234.

[7] R. Mathon, A. Rosa: $2-(v, k, \lambda)$ designs of small order. The CRC handbook of combinatorial designs, Ed. J. Colburn, J. Dinitz, CRC, (1996), 3-41.

[8] L. Milazzo, Zs. Tuza: Upper chromatic number of Steiner triple and quadruple systems. Discrete Math. 174 (1997), 247-259.

[9] L. Milazzo: On upper chromatic number for SQS(10) and SQS(16). Le Matamatiche (1995), L, 179-193.

[10] L. Milazzo: Sul numero cromatico superiore nei sistemi di Steiner. PhD Thesis, University of Catania, (1996).

[11] V. I. Voloshin: The mixed hypergraph. Comput. J. Moldova 1 (1993), 45-52.

[12] V. I. Voloshin: On the upper chromatic number of a hypergraph. Australasian J. Combin. 11 (1995), 25-45. 